First EMC3 El RENE Si mul at i ons wi th Di vert or Legs of LHD in Real i stic Devi ce Geometry

\begin{tabular}{|l|l|}
\hline $\begin{array}{l}\text { j our nal or } \\
\text { publ i cat i on } \mathrm{t} \text { i t l e }\end{array}$ & Cont r i but i ons to PI asma Physi cs \\
\hline vol une & 54 \\
\hline number & $4-6$ \\
\hline page range & $437-441$ \\
\hline year & 2014 - 06- 20 \\
\hline URL & ht t p: //hdl . handl e. net /10655/00012663 \\
\hline
\end{tabular}




\title{
First EMC3-EIRENE simulations with divertor legs of LHD in realistic device geometry
}

\author{
Gakushi Kawamura ${ }^{1, *}$, Yuhe Feng ${ }^{2}$, Masahiro Kobayashi ${ }^{1}$, Mamoru Shoji ${ }^{1}$, Tomohiro \\ Morisaki $^{1}$, Suguru Masuzaki ${ }^{1}$, and Yukihiro Tomita ${ }^{1}$ \\ 1 National Institute for Fusion Science, 322-6 Oroshi-cho Toki, Gifu 509-5292, Japan \\ ${ }^{2}$ Max-Planck-Institut für Plasmaphysik, Wendelsteinstraße 1 D-17491 Greifswald, Germany
}

Received XXXX, revised XXXX, accepted XXXX

Published online XXXX

Key words simulation, transport, neutral, recycling, divertor, LHD

An extended mesh system for EMC3-EIRENE has been developed to simulate peripheral plasma including the ergodic and the divertor leg regions of LHD. Both the open and the closed divertor configurations are available. A series of simulations for $8 \mathrm{MW}$ input power, five different electron densities at the LCFS (last closed flux surface) and the open/closed configurations were carried out. Approximately 10 times larger neutral pressure was observed under the dome structure compared with the open configuration, which is in good agreement with experimental measurements. In the case of the closed configuration, the leg regions have a large contribution of ionization to hydrogen recycling. In the case of high density discharges, however, electron temperature in the legs becomes low and the major contribution of ionization moves to the ergodic region. Significant influence of configurations is observed in the inboard side of LHD, where closed divertor components are installed but little influence is seen near the LCFS.

Copyright line will be provided by the publisher

\section{Introduction}

Transport simulation is one of essential tools to understand particle and energy confinement in fusion devices. Recent development of simulation techniques and large parallel computers reduce difficulty of the three-dimensional simulation of helical plasmas. As for Large Helical Device (LHD) [1], three-dimensional fluid code, EMC3EIRENE [2-4], was applied to the simulation of the ergodic region, which has stochastic magnetic field and involves both perpendicular and parallel transport. Validity of EMC3-EIRENE focusing on plasma and impurity transport in the ergodic region of LHD has been confirmed [4-7]. Divertor-leg plasma was, however, excluded to reduce technical difficulties arising from making a calculation mesh on the outer region of plasma. Simulation without a divertor leg leads to different global transport of hydrogen and impurities near divertor plates and that can be significant in the case of the closed divertor configuration introduced recently in LHD to make efficient particle control by pumping of hydrogen gas. Although increase of neutral gas pressure in the closed configuration was confirmed by simulation [8] and experiment [9] in good agreement, plasma transport simulation was limited to open divertor configuration without the legs.

Extension of the calculation mesh of EMC3-EIRENE code to the divertor legs of LHD has been achieved and simulation study on plasma-neutral interactions in realistic geometry is presented in this paper. After the introduction in Sec. 1 the development of simulation model is described in Sec. 2. The simulation results and discussions of plasma-neutral interactions are presented in Sec. 3. Finally they are summarized in Sec. 4.

\section{Simulation model and calculation mesh system for LHD}

The magnetic field of LHD has $n=10$ helical periodicity. Plasma distribution on the poloidal cross-sections at toroidal angle $\phi=0^{\circ}$ and $18^{\circ}$, so called "vertically elongated plane" and "horizontally elongated plane" respectively, has up-down symmetry. Contour plots of connection length on poloidal cross-sections are given in

\footnotetext{
* Corresponding author E-mail: kawamura.gakushi, Phone: +81 572582279 , Fax: +81 572582630
} 


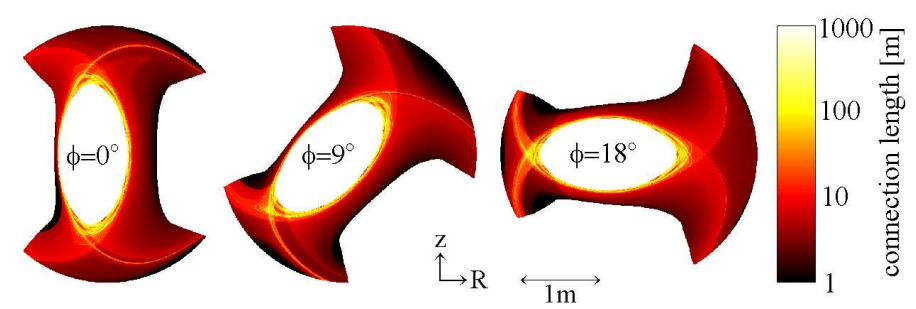

Fig. 1 Contour plots of connection length on poloidal cross-sections at the toroidal angle $\phi=0,9$ and $18^{\circ}$. The magnetic axis is at $R=3.6 \mathrm{~m}$. Center of the device is located at the left-hand side of each plot. KMAG code [11] is used to calculate them. Flux tubes with long connection length transport large particle and heat flux.

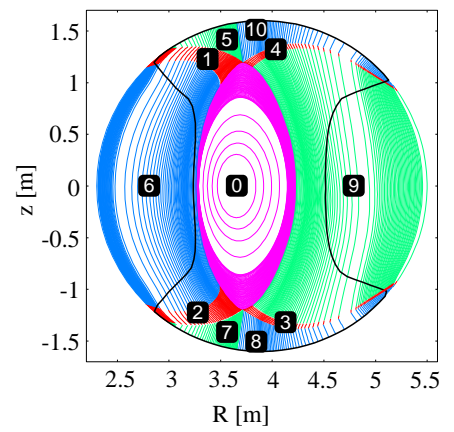

Fig. 2 Meshes on $\phi=0^{\circ}$ plane. The number on each mesh represents a zone number.

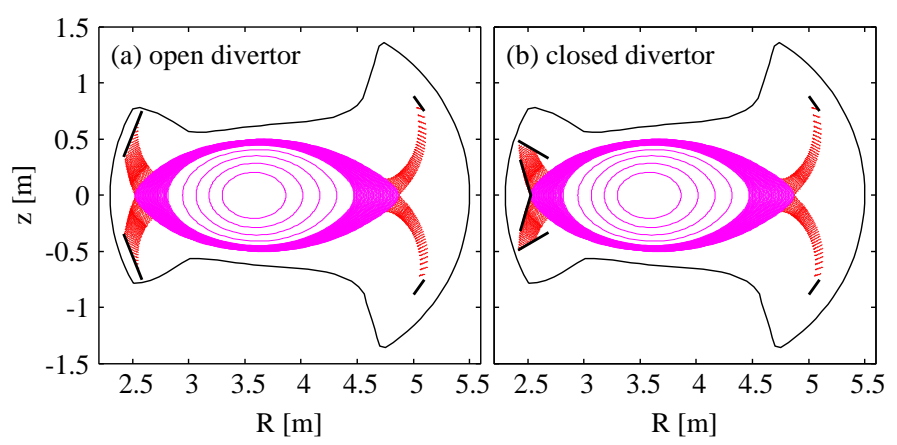

Fig. 3 Calculation meshes of plasma and divertor plates on $\phi=18^{\circ}$ plane; (a) open and (b) closed divertor configuration.

Fig. 1. The simulation box includes an $18^{\circ}$ toroidal section, which corresponds to the full-torus with the aid of the up-down symmetry and the helical periodicity. In order to ensure up-down symmetry at the two end planes, we made two block of meshes in ranges $\phi=0-9^{\circ}$ and $\phi=9-18^{\circ}$. Physical quantities are mapped with each other on the $\phi=9^{\circ}$ plane. That concept of mesh system has been validated in the previous EMC3-EIRENE simulations [4].

The code solves Braginskii-type [10] two-fluid equations along flux tubes with perpendicular transport of particle and heat modeled by diffusive transport coefficients, $D$ and $\chi\left[\mathrm{m}^{2} / \mathrm{s}\right]$, respectively. The heat transport coefficient for ion and electron are assumed to be the same in this paper. The code uses field-aligned meshes, where an index of grid points is labeled along a magnetic field line, to realize fast integration along field lines and small aliasing error arising from finite resolution of meshes. However it is difficult to make a single mesh covering the whole plasma because cells defined by two meshes of neighboring toroidal angles is too much distorted by large magnetic shear inside a divertor section. In extreme cases, a magnetic field line traced from a certain point in a leg is wound around a helical coil beyond the vacuum vessel.

We divide plasma into an ergodic region and four legs to reduce influence of distortion due to magnetic shear. The rest of regions is covered by vacuum meshes. Each region has a "zone number" shown in Fig. 2, i.e. 0: ergodic region, 1-4: leg regions and 5-10: vacuum regions. Neutral particles are traced in all zones, and plasma is solved in zones $0-4$. The core region, which corresponds to the sparse mesh in the center of zone 0 , is also excluded from the calculation of plasma. Meshes in the other block, i.e. $\phi=9-18^{\circ}$, has the zone number $12-21$. The outermost boundary is shaped into a circle with radius of $1.6 \mathrm{~m}$. The vacuum vessel, which is drawn as a black curve in the figure, is installed independently of meshes. Divertor plates shown in Fig. 3 are also installed as additional walls. Only grid points of legs inside the plates are used for calculation. Resolution of toroidal angle is determined to resolve the shape of legs near divertor plates and to avoid strong distortion. We use $0.25^{\circ}$ as toroidal resolution, which requires $(9 / 0.25+1) \times 2=74$ poloidal planes.

We developed the mesh system for inward shifted configuration, i.e. magnetic axis is at $R=3.6 \mathrm{~m}$. The first step of mesh generation is calculation of connection length on poloidal planes at $\phi=0,9$ and $18^{\circ}$ like Fig. 1 , with the aid of a magnetic-field-line tracing code, KMAG [11]. The second step is generation of mesh covering 

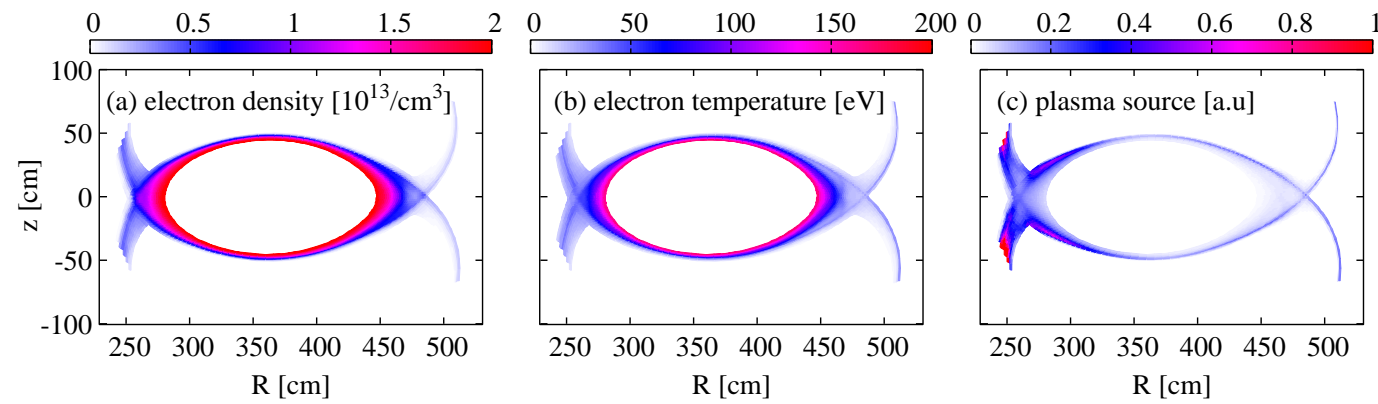

Fig. 4 Contour plot of (a) electron density, (b) electron temperature and (c) plasma source on the plane $\phi=18^{\circ}$ with open divertor configuration.

the ergodic region on $\phi=0$ and $18^{\circ}$ planes. A Poincare plot of magnetic-field-lines gives flux surfaces inside the LCFS (last closed flux surface). Since points in the plot in the ergodic region become chaotic but still have structure of bands except at the vicinity of the plasma surface, we employed the bands as guides to determine the grid points. After making meshes on the planes at $\phi=0$ and $18^{\circ}$, meshes on the other 72 planes were generated by KMAG code. The third step is generation of legs. Meshes of four legs on $\phi=9^{\circ}$ plane were constructed to cover area of long connection length, roughly more than $10 \mathrm{~m}$, and then meshes on other 73 planes were made by KMAG code. On $\phi=0$ and $18^{\circ}$ planes, the legs were flipped upside down to ensure the symmetry. In order to avoid winding motion of the leg during magnetic-field tracing, the last grid points of legs were restricted on the $R=1.6 \mathrm{~m}$ circle. Although that artificial adjustment causes narrow regions which is invalid for calculation because of wrong magnetic field such as $\nabla \cdot \vec{B} \neq 0$, they are behind the divertor plates and do not affect the plasma. The last step is generation of vacuum meshes. We developed automated tools to make vacuum meshes. They are not restricted by magnetic field and were made by purely geometrical means.

\section{Simulation results and discussions}

We carried out an EMC3-EIRENE simulation with input power: $P=8 \mathrm{MW}$, electron density at the LCFS: $n_{\mathrm{LCFS}}=2 \times 10^{13} / \mathrm{cm}^{3}$. The perpendicular transport coefficients are assumed as follows; $D=1 \mathrm{~m}^{2} / \mathrm{s}, \chi=3 \mathrm{~m}^{2} / \mathrm{s}$. Simulation results of the open configuration are shown in Fig. 4. Plasma profiles are smoothly connected across zones. No numerical instability was caused by the new mesh system and calculation time was not significantly larger than the previous mesh system. Electron density and temperature reflect the connection length distribution. Ions arriving at divertor plates recombine with electrons and are released as neutral gas. They move independently of magnetic field and are ionized in the plasma. Distribution of the plasma source in Fig. 4(c) reflects those processes and consistent with observation of $\mathrm{H}_{\alpha}$ radiation [12].

The most obvious difference measured in experiment between open and closed divertor configurations is the increase of neutral pressure under the dome structure [9]. In order to simulate that, we carried out a series of simulations with constant input power $P=8 \mathrm{MW}$ and different density $n_{\mathrm{LCFS}}=1,2,4,6$ and $8 \times 10^{13} / \mathrm{cm}^{3}$. In Fig. 5, neutral pressure measured in experiments and simulations is given. The pressure of the closed configuration is approximately $10 \sim 20$ times higher than that of the open configuration. Simulation results show good agreement with measurements $[9,13]$. The dependence of pressure on electron density at the LCFS is the same as measurements. Those results support the validity of EMC3-EIRENE simulation with the new mesh system. Prediction of the absolute value of pressure is, however, difficult because the pressure involves uncertainties by the factor of $2 \sim 3$. Higher neutral temperature on release from walls gives higher pressure. Another set of simulations suggests that a half $D$ and $\chi$ leads to approximately a half neutral pressure. Contribution of hydrogen atoms, which have higher temperature, to a pressure gauge is another uncertainty.

The increase of neutral pressure is caused by not only geometrical effect of the dome, but also by an increase of plasma-neutral interaction, i.e. recycling. Figure 6 shows number of hydrogen atoms ionized in the plasma per second. 'Total' in the figure means the amount in whole plasma and 'leg' means the amount only in legs. Since there is no additional source such as gas puffing or sink such as vacuum pumping, all of hydrogen is recycled in the simulation. Therefore, the total plasma source increases according to electron density at the LCFS. The 

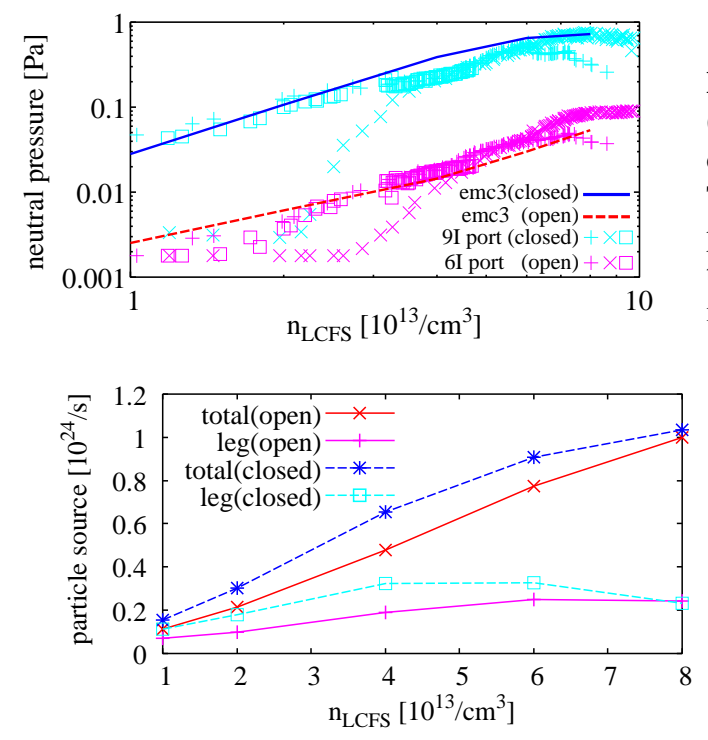

Fig. 6 Plasma source in whole plasma region and only in legs.
Fig. 5 Comparison of neutral pressure between simulation (solid line) and measurements (dots). Three symbols of dots correspond to the shot number 99368, 99370 and 102301. The both axes are in logarithmic scale. Constant neutral temperature, $500^{\circ} \mathrm{K}$, is assumed to calculate pressure from neutral density given by simulations. The horizontal axis, $n_{\mathrm{LCFS}}$, is electron density at the LCFS. The input power is fixed.

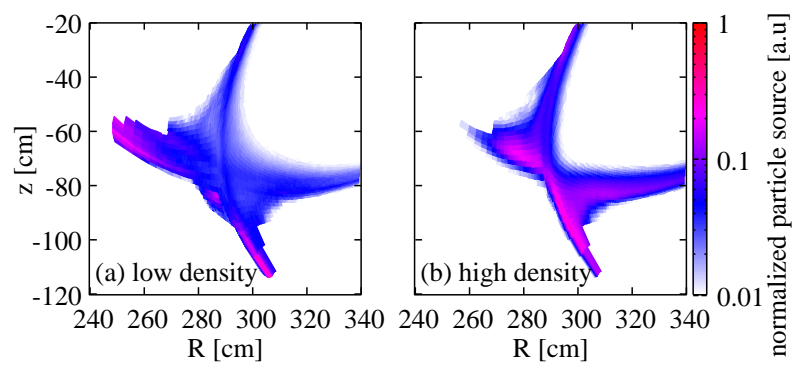

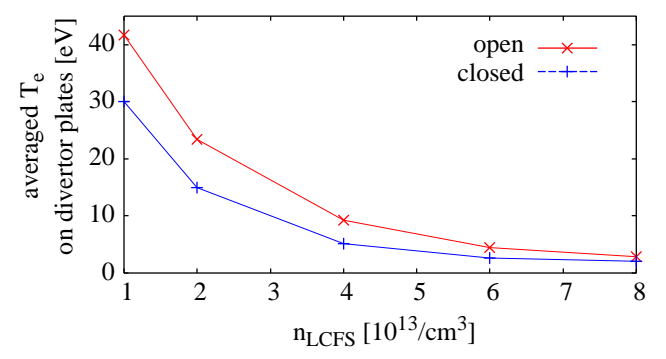

Fig. 7 Electron temperature average over all the plasma-wetted area with weight of plasma pressure.

Fig. 8 Contour plots of normalized plasma source at inner divertor on $\phi=9^{\circ}$ plane; (a) low density, $n_{\mathrm{LCFS}}=2 \times 10^{13} / \mathrm{cm}^{3}$, and (b) high density, $n_{\mathrm{LCFS}}=$ $8 \times 10^{13} / \mathrm{cm}^{3}$. The simulations are carried out in the closed configuration.

change from open to closed configuration increases the plasma source. In the case of low density discharges, $n_{\mathrm{LCFS}}<5 \times 10^{13} / \mathrm{cm}^{3}$, the amount of increase from open to closed configuration are almost the same in both total and leg. In the case of high density discharges, the increase of the source in the legs becomes small and fraction of the source outside the legs increases significantly. Those results imply that increase of recycling is caused mainly in the leg regions in low density cases but in the ergodic region in high density cases. At high densities, the relative source rates in the legs decreases with increasing density. This is related to the electron temperature in the legs. Since input power is fixed to $8 \mathrm{MW}$, increase of electron density causes decrease of electron temperature and hence low ionization rate. Figure 7 shows averaged electron temperature on the divertor plates in open and closed configurations. In the case of high density, $n_{\mathrm{LCFS}}>5 \times 10^{13} / \mathrm{cm}^{3}$, and closed configuration, average electron temperature becomes too low for hydrogen atoms to ionize. Transition of dominant region where ionization takes place is clearly seen in Fig. 8. Similar transition is observed also in the case of open configuration. The fraction of plasma source in the ergodic region is larger than that in the closed configuration even in the low density discharge. Therefore the change in the transition is weaker than that in the closed configuration. That difference is caused by the large amount of the neutral transport toward the ergodic region because the most of the neutrals generated on the surface can go through the leg before ionized, see Fig. 3.

In order to study influence of divertor configurations on plasma parameters, we compare radial electron temperature distribution along the mid-plane in Fig. 9. In the center region, i.e. $270 \mathrm{~cm}<R<460 \mathrm{~cm}$, difference between open and closed configuration is small. A clear difference is observed on the inboard side, $R<270 \mathrm{~cm}$, where electron temperature becomes significantly low in the case of closed configuration. On the outboard side, difference of temperature is relatively small. Although figure is not shown here, electron density has the opposite dependence on configuration and the same dependence on the location. The differences between inner and outer divertor come from plasma-neutral interaction, which takes place much stronger on the inboard side. 

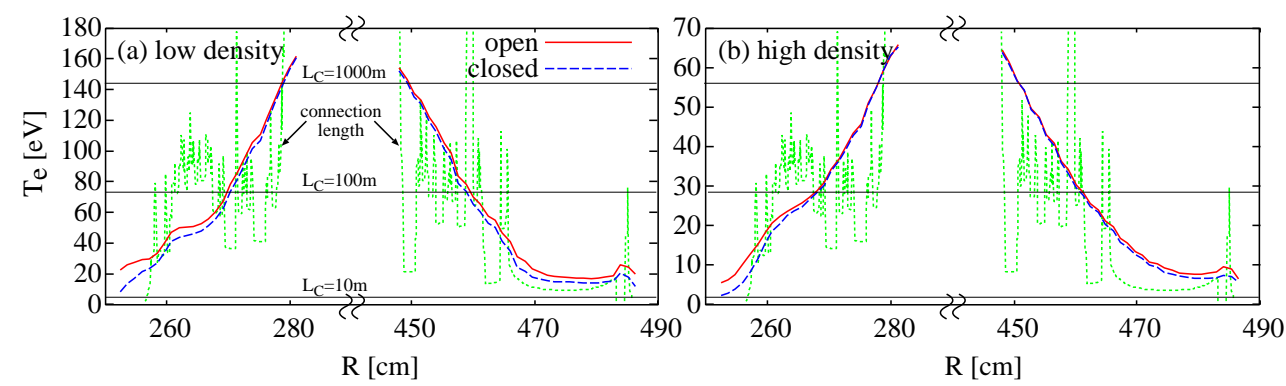

Fig. 9 Electron density distribution along the mid-plane on $\phi=18^{\circ}$ plane, where Thomson scattering measurements are performed; (a) low, $2 \times 10^{13} / \mathrm{cm}^{3}$, and (b) high, $6 \times 10^{13} / \mathrm{cm}^{3}$, density. Connection length, $L_{\mathrm{C}}$, is drawn in logarithmic scale.

\section{Conclusions}

An extended mesh system of EMC3-EIRENE has been developed to simulate peripheral plasma of LHD. The new mesh system covers the ergodic and the divertor leg regions. Major differences from the previous mesh system are addition of the leg regions and higher toroidal resolution. Both the open and the closed divertor configurations are now available. Calculation time was not significantly larger than the previous mesh system.

We carried out series of simulations for 8MW input power, five different electron densities at the LCFS and the open and the closed configurations. From simulation results we investigated the difference of plasma-neutral interaction between the open and the closed configuration. First, concerning neutral transport, approximately 10 times larger pressure was observed under the dome structure compared with the open configuration. The compression ratio and dependence of the pressure on the electron density at the LCFS show a good agreement with experimental measurements. Second, difference of recycling between configurations was elucidated. In the case of low density discharges, $n_{\mathrm{LCFS}}<5 \times 10^{13} / \mathrm{cm}^{3}$, ionization fraction in the leg region becomes large by changing from open to closed configuration. In the case of high density discharges, the increase of ionization in legs are relatively small and that in the ergodic region is the major contribution to the total plasma source. Third, influence of configurations on plasma profiles were analyzed. Electron temperature and density in center region, i.e. $270 \mathrm{~cm}<R<460 \mathrm{~cm}$, suffer no clear influence but outer region, especially inboard side where closed divertor components are installed, have significant changes; lower electron temperature and higher electron density.

From the above results, we confirmed the validity of the extended EMC3-EIRENE simulation with the new mesh system and the usefulness to analyse transport and interactions between plasma and neutrals. Detailed comparison with measurements and further investigation of transport are necessary to understand perpendicular and parallel transport of plasma. Analysis of impurity transport in the closed configuration is another target.

Acknowledgements The authors acknowledge support by the LHD experiment group. This work was done under IEA contract for TEXTOR cooperation and was supported partly by a Grant-in-Aid for Young Scientists (B) of Japan Society for the Promotion of Science (No. 24760702) and performed with the support and under the auspices of the NIFS Collaboration Research program (NIFS13KNST055).

\section{References}

[1] N. Oyabu, et al., Nucl. Fusion 34, 387 (1994).

[2] Y. Feng et al., Contrib. Plasma Phys. 44, 57 (2004).

[3] D. Reiter et al., Nucl. Fusion 47, 172 (2005).

[4] M. Kobayashi et al., Fusion Sci. Technol. 58, 220 (2010).

[5] M. Kobayashi et al., J. Nucl. Mater. 390-391, 325 (2009).

[6] M. Kobayashi et al., J. Nucl. Mater. 363-365, 294 (2007).

[7] M. Kobayashi et al., Nucl. Fusion 53, 033011 (2013).

[8] M. Shoji et al., Nucl. Mater. 390-391, 490 (2009).

[9] S. Masuzaki et al., Plasma Fus. Res. 6, 1202007 (2011).

[10] S.I. Braginskii, Rev. Plasma Phys. 1, 205 (1965).

[11] Y. Nakamura et al., J. Plasma Fusion Res. 69, 41 (1993).

[12] M. Goto and S. Morita, Rev. Sci. Instr. 77, 10F124 (2006).

[13] T. Morisaki et al. Nucl. Fusion 53, 063014 (2013). 